\title{
Review
}

\section{Hyperinsulinaemia and hyperglycaemia: possible risk factors of colorectal cancer among diabetic patients}

\author{
C. K. Chang ${ }^{1,2}$, C. M. Ulrich ${ }^{1,2}$ \\ ${ }^{1}$ Department of Epidemiology, School of Public Health and Community Medicine, University of Washington, Seattle, USA \\ ${ }^{2}$ Epidemiology Program, Fred Hutchinson Cancer Research Center, Seattle, USA
}

\begin{abstract}
Hyperinsulinaemia and hyperglycaemia are two possible risk factors for colorectal cancer, which constitutes the third leading cause of cancer death in Western countries. Molecular evidence as well as animal models provide support for these associations: Insulin has been shown to be an important growth factor for colonic carcinoma cells, and both insulin and insulinlike growth factor-1 receptors have been detected in colon cancer tissue. The insulin-signal transduction pathway is involved in the regulation of gene expression and apoptosis. The role of hyperglycaemia in carcinogenesis could include pathways via luminal factors (related to fecal bile acid concentrations, stool bulk, and prolonged transit time) or circulatory factors (via glucose as the only energy source for neoplastic cells). This review summarizes the epidemiologic lit-
\end{abstract}

erature with respect to hyperinsulinaemia and hyperglycaemia as risk factors for colorectal cancer, and aims to integrate the biological and epidemiological evidence. Epidemiologic findings to date indicate a slightly increased risk of colorectal cancer for diabetic patients; however, there are some inconsistencies. Possible explanations for these inconsistencies include inadequate information about patients' diabetic disease and treatment states. We suggest that future studies should take medical history, staging and treatment for hyperinsulinaemia and hyperglycaemia into account to further our understanding of the role of hyperglycaemia and hyperinsulinaemia in colorectal carcinogenesis. [Diabetologia (2003) 46:595-607]

Keywords Diabetes mellitus, colorectal cancer, carcinogenesis, hyperglycaemia, hyperinsulinaemia, insulin resistance, IGF-1, epidemiology, review.
Colorectal cancer is a major public health problem, being the fourth most common cancer and the second most common cause of cancer death in the USA [1]. On an international scale, colorectal carcinoma ranks second in cancer incidence in the majority of devel-

Received: 10 February 2003 / Revised: 19 March 2003

Published online: 23 May 2003

(C) Springer-Verlag 2003

Corresponding author: Dr. C. M. Ulrich, Cancer Prevention Research Program, Fred Hutchinson Cancer Research Center, PO Box 19024, MP-900, Seattle, WA 98109, USA

E-mail: nulrich@fhcrc.org

Abbreviations: DCCT, Diabetes Control and Complications Trial; IGFBP-3, IGF binding protein-3; NSAIDs, Nonsteroidal anti-inflammatory drugs; OTC, Over the counter; UKPDS, United Kingdom Prospective Diabetes Study. oped countries with high-risk areas being North America, Western Europe, and New Zealand [2]. Risk factors for colorectal cancer or polyps include meat consumption, smoking, alcohol consumption, and a positive family history. Inverse associations exist with physical activity, vegetable or folate intake, and use of nonsteroidal anti-inflammatory drugs (NSAIDs) or hormone replacement therapy [3].

The digestive system supports an individual's basic physical needs for nutrition and energy. Glucose is one of the prime biochemical entities used for transportation, storage, and generation of energy. The blood glucose concentration is kept in homeostasis through a complex network of energy output, dietary intake and hormonal regulation, involving insulin, glucagon, epinephrine, growth hormone, and cortisol. If this homeostasis is disturbed, a series of pathoglycaemic disorders 
can develop [4]. Insulin plays a key role in this regulatory process, with a concentration in healthy subjects of 0.4 to $0.8 \mathrm{ng} / \mathrm{ml}$ after an overnight fast. Abnormalities of insulin concentrations can be caused by autoimmune-mediated beta cell failure in the pancreas (Type 1 diabetes) or by insulin resistance (Type 2 diabetes). Type 1 diabetes mellitus arises in the early stage of life, while Type 2 diabetes mellitus is a degenerative disorder among older people with complex causes [5]. In Type 1 diabetes mellitus patients, insulin secretion decreases continuously along with the development of diabetes, and then induces hyperglycaemia when insulin secretion is insufficient. At the early stage of Type 2 diabetes, hyperinsulinaemia exists because of a compensatory response to insulin resistance, whereas in the later stages, beta-cell malfunction contributes to a hypoinsulinaemic response as diabetes mellitus progresses $[5,6]$. Without proper control of hyperglycaemia, diabetic patients are at risk of cardiovascular disease, hypertension, retinopathy, nephropathy, neuropathy, dyslipidemia, and ketoacidosis [7]. Diabetes mellitus has become a crucial health issue with major public health impact all over the world [8].

Previous reviews on the link between hyperglycaemia, hyperinsulinaemia and colorectal cancer risk have focused on the aspects of underlying biological models $[9,10,11,12,13]$, animal models [11], and some epidemiologic evidence $[9,12,13,14,15]$. The current review centres on a comprehensive summary of the epidemiological literature on diabetes and colorectal cancer risk, thus describing one important line of evidence regarding the insulin-colorectal cancer hypothesis. Suggestions are made regarding study designs addressing limitations inherent in previous studies that could have obscured the associations between hyperinsulinaemia, hyperglycaemia, and colorectal cancer.

\section{Evidence linking hyperinsulinaemia and hyperglycaemia to colorectal cancer}

Several risk factors observed in epidemiologic studies provide a link to hyperinsulinaemia or hyperglycaemia. Those include a "Western Lifestyle" with diets low in fruits and vegetables or fibre, and high in fat and cooked meat, restricted physical activity, and an increased BMI [3]. In molecular and animal studies, hyperinsulinaemia [10] and hyperglycaemia [9] have been shown to be independent risk factors of colorectal carcinogenesis. In the following sections, we briefly describe the proposed biological mechanisms.

\section{Biological evidence supporting an association between hyperinsulinaemia and colorectal cancer}

The biological mechanisms for insulin, insulin-like growth factors (IGF) and colon cancer have been ex- tensively described [15]. Briefly, in vitro, insulin is an important growth factor for colonic mucosal cells and acts as a mitogen for colonic carcinoma cells [16, 17, 18]. Insulin-like growth factor-1 (IGF-1) inhibits apoptosis and is required for cell cycle progression [19]. Both normal colorectal epithelium and colon cancer tissue have insulin and IGF-1 receptors [20, 21]; upon activation by IGF-1, the receptor-ligand complex inhibits apoptosis with continuation of the cell cycle $[16,17,18,21]$. Therefore, both pre-maligant and cancerous stages can be affected by IGF-1. IGF-1 could also enhance the production of vascular endothelial growth factor which is essential for angiogenesis and tumour growth [22]. IGF-2 can stimulate IGF-1 receptors, and colorectal tumours show increased concentrations of IGF-2 mRNA and secreted peptide $[23,24,25]$. The insulin-signal-transduction pathway can be mediated by activation of the PI3 kinases or by ras gene activation and is involved in the regulation of gene expression and mitogenicity [26, $27,28]$. Activating ras mutations occur in about half of colonic cancers [29]. Recently, positive associations between the plasma concentration of IGF-1 [30, $31]$ or concentrations of circulating C-peptide (a surrogate marker for insulin secretion) and colorectal cancer have been observed [30]. These findings were extended by showing that high concentrations of circulating IGF-1 and very low concentrations of insulinlike growth factor binding protein-3 (IGFBP-3) were associated independently with an increased risk of advanced colorectal adenomas and cancer [32].

The potential biological mechanisms linking insulin resistance to colorectal cancer by influencing cell growth and cell cycle control have been well described [11]. Nevertheless, it is possible that a particular factor could affect colon cancer risk both by influencing insulin concentrations and through other mechanisms $[10,11]$.

\section{Biological evidence supporting an association between hyperglycaemia and colorectal cancer}

There are at least two possible biological mechanisms linking hyperglycaemia to colorectal cancer. Among patients with diabetes, higher levels of fecal bile acid concentrations, greater stool bulk, and longer transit times have been observed [33]. It has been hypothesized that these luminal factors are involved in the carcinogenesis of the colorectal mucosa $[34,35]$. In particular, fecal bile acids have been shown to promote colorectal neoplasia in animal models [36, 37, 38]. However, associations between stool transit time and colorectal cancer [39] or adenomatous polyps [40] were not observed in humans. This association warrants further investigation. Secondly, the energy supply to neoplastic cells might be important: In contrast to the normal colonic epithelium which is capable of 
both aerobic and anaerobic metabolism, using short chain fatty acids, amino acids, and glucose as fuel [41], neoplastic cells switch from aerobic to anaerobic metabolism, requiring glucose rather than fatty acids for fuel [42]. Thus, plasma glucose might be associated with colorectal neoplasia by acting as a direct energy source to the tumour.

Animal studies investigating diets high in sucrose and low in dietary fibre support a positive association with colonic cell proliferation and growth rates of aberrant crypt foci $[43,44,45,46]$. However, there is some, but insufficient, evidence regarding the relationship between colorectal cancer and plasma glucose $[47,48]$ or sugar intake [49] in humans.

\section{Epidemiological studies investigating associations between hyperglycaemia or hyperinsulinaemia and colorectal cancer}

Epidemiological studies of various designs have investigated associations between hyperglycaemia or hyperinsulinaemia and colorectal cancer risk. These studies include: (i) case-control and cross-sectional studies of patients with colorectal cancer or adenomas compared to appropriate control group(s) investigating exposures as a history of diabetes or impaired glucose tolerance; (ii) cohort studies of patients with diagnosed diabetes and subsequent risk of colorectal cancer; (iii) cohort studies using baseline measures for tracing insulin resistance or underlying diabetes and subsequent risk of colorectal cancer among general populations.

\section{Case-control and cross-sectional studies}

In the past two decades, several case-control or crosssectional studies on the risk of colorectal cancer associated with diabetes have been conducted (Table 1), mostly reporting either no association or moderate positive associations: An approximately two-fold risk of overt diabetes has been reported in a comparison of colon/rectum cancer cases to lung cancer or hip fracture cases (hospital based) [50]. In a large multi-site cancer case-control study, prior history of diabetes was not found to be a risk factor for colorectal cancer compared to hospital controls [51]. Kune et al. examined several chronic illnesses as risk factors of colorectal cancer, without observing an increased risk associated with diabetes [52]. A series of case-control studies conducted in Northern Italy reported either no association, or a moderate positive association between Type 2 diabetes and risk of colorectal cancer $[53,54,55]$. However, a population-based case-control study conducted in Hawaii showed increased odds ratios (OR) for distal colorectal cancer among patients with diabetes $(\mathrm{OR}=1.9,95 \% \mathrm{CI}=1.1-3.5$ for men;
$\mathrm{OR}=3.0,95 \% \mathrm{CI}=1.2-7.1$ for women) [56]. A recent report from Switzerland showed no substantial change in risk of colorectal cancer among men reporting a history of diabetes $(\mathrm{OR}=1.3,95 \% \mathrm{CI}=0.6-2.7)$, but a significantly increased risk among women $(\mathrm{OR}=3.6$, $95 \% \mathrm{CI}=1.1-12.1)$. The number of patients reporting a history of diabetes was small (21 cases and 18 controls), but the risk was substantially increased for individuals diagnosed with diabetes for five or more years (OR=4.1, 95\% CI=1.6-10.8) [57].

Limitations of these studies were uncontrolled confounding factors [50], the use of hospital-based cases and controls [50,51, 53, 54, 55, 57], and reliance on self-report for diabetes history [51, 52, 53, 54, 55, 57]. Lack of controlling for confounding factors could distort the associations between diabetes history and colorectal cancer. Hospital-based studies can result in a biased selection of control patients, in that they would not represent the general characteristics of the population where they come from. The United Kingdom European Prospective Investigation into Cancer was analyzed cross-sectionally for self-reported diabetes (Type 2 diabetes mellitus mostly) and prevalent colorectal cancer. The proportion of colorectal cancer cases with diabetes was $6.1 \%$, whereas only $1.9 \%$ were observed among controls $(\mathrm{OR}=2.9$, 95\% $\mathrm{CI}=1.5-5.8$ ) [58]. However, it is possible that a higher diagnosis rate for colorectal cancer among cases could have contributed to this increased risk.

Several recent studies used biomarkers associated with hyperglycaemia or hyperinsulinaemia, rather than the rather imprecise exposure measure of selfreported diabetes mellitus history. Kono et al. measured glucose tolerance status in Japanese men aged 48 to 59 years who received colorectal cancer screening by sigmoidoscopy as part of a pre-retirement health examination [59]. Glucose-tolerance status was used to classify participants as "normal", "having impaired glucose tolerance", "newly diagnosed Type 2 diabetes", and "diabetes under treatment". Adenoma risk was significantly increased among participants with newly diagnosed Type 2 diabetes $(\mathrm{OR}=1.4$, $95 \% \mathrm{CI}=1.0-2.0$, adjusted for possible confounders). Of twenty-one individuals in the "diabetes under treatment" category, all showed a non-significant risk of similar magnitude (adjusted $\mathrm{OR}=1.3,95 \%$ $\mathrm{CI}=0.8-2.2$ ). Nevertheless, the cross-sectional nature of this study limits the conclusions that can be drawn. Another screening study in a similar setting showed no increased risk of adenomas among those with impaired glucose tolerance, but an increased risk associated with Type 2 diabetes mellitus ( $\mathrm{OR}=2.2,95 \%$ $\mathrm{CI}=1.1-4.0$, adjusted for age and BMI) [60]. Overall, the results from case-control and cross-sectional studies on diabetes history provide only modest support to the hypotheses of hyperinsulinaemia and hyperglycaemia as risk factors for colorectal cancer. Studies of biomarkers associated with hyperinsulinaemia or 
Table 1. Case-control and cross-sectional studies relating diabetes history or glucose intolerance to colorectal cancer or adenomas

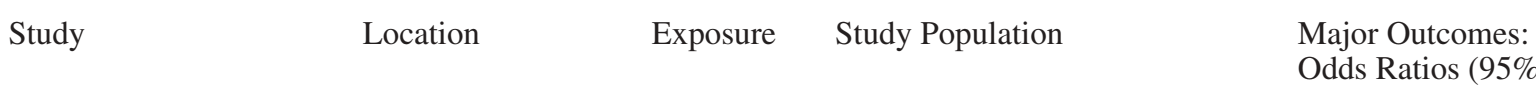

Odds Ratios (95\% CI)

Case-control Studies:

Williams et al. (1984)

[50]

Mississippi, USA

Diabetes history

O’Mara et al. (1985) [51]

\section{Buffalo, NY,} USA

Diabetes history

Kune et al. (1988)

[52]

La Vecchia et al. (1991)

[53]

La Vecchia et al. (1994) [54]

La Vecchia et al. (1997) [55]

Le Marchand et al.

(1997) [56]

Hawaii, USA

Diabetes history

Melbourne

Diabetes Australia history

Greater Milan area, Italy

Diabetes history

Greater Milan area, Italy

Six areas in northern, central, and southern Italy

Diabetes history

Diabetes history

Levi et al. (2002)

[57]
Canton of Vaud, Diabetes Switzerland history and 252 rectal cancer cases from three medical centres Controls: 1916 admitted lung cancer and 1662 hip fracture inpatients from the same hospitals

Cases: 335/295 white females \& 277/379 males with colon/ rectal cancers

Controls: 2475 white females \& 2363 males admitted without neoplastic conditions

Cases: 715 colorectal cancers Control: 727 age/sex matched controls in community

Cases: 673 colon cancers

\& 405 rectal cancers

Controls: 1501 hospital controls admitted for acute, nonneoplastic, non-digestive tract conditions

Cases: 828 colon cancers \& 498 rectal cancers Controls: 7834 hospital controls admitted for acute, nonneoplastic, non-metabolic, non-hormone-related conditions

Cases: 1225 colon cancers

\& 728 rectal cancers

Controls: 4154 hospital controls admitted for acute, nonneoplastic, non-digestive tract conditions and required no longterm modification of diet from the same surveillance areas

Cases: 698 male and 494 female colorectal cancers

Controls: 1192 age/sex/ethnicitymatched community residents
Cases: 696 colon cancer cases,

Compared to lung cancer cases ${ }^{(\mathrm{C})}$ : Colon: 2.0 (1.5-2.8)

Rectum: 1.8 (1.1-2.9)

Compared to hip fracture cases $(\mathrm{C})$ :

Colon: 2.3 (1.6-3.2)

Rectum: 2.0 (1.2-3.3)

Males(AS):

Colon: 1.2

Rectum: 1.1

Females $^{(\mathrm{AS})}$ :

Colon: 1.4

Rectum: 1.0

(95\% CIs not provided; all non-significant)

Total $^{(\mathrm{AS})}$ : $1.0(0.6-1.7)$

Males(AS): 1.3 (0.7-2.5)

Females $^{(\mathrm{AS})}$ : 0.8 (0.4-1.6)

Colon$^{(\mathrm{MV})}: 1.7(1.1-2.5)^{\mathrm{a}}$

$\operatorname{Rectal}^{(M V)}: 1.5(1.0-2.5)^{\mathrm{a}}$

Colon(AS): $^{(1.1}(0.8-1.5)$

Rectal $^{(\mathrm{AS})}$ : 0.9 (0.6-1.3)

Total(MV): $1.3(1.0-1.6)^{\mathrm{b}}$

Colon$^{(\mathrm{MV})}$ : $1.2(0.8-1.6)^{\mathrm{b}}$

$\operatorname{Rectal}^{(\mathrm{MV})}: 1.5(1.1-2.2)^{\mathrm{b}}$

Colorectal cancer(MV):

Males: $1.4(1.0-1.9)^{\mathrm{b}}$

Female: $1.2(0.8-1.8)^{\mathrm{b}}$

Males(MV):

Total: $1.2(0.8-1.7)$

Proximal colon: $1.0(0.4-1.8)$

Distal colon: 1.9 (1.1-3.5)

Rectum: 0.7 (0.3-1.6)

Females $^{(\mathrm{MV})}$.

Total: 1.8 (1.1-2.8)

Proximal colon: $1.3(0.6-2.7)$

Distal colon: 3.0 (1.2-7.1)

Rectum: 1.7 (0.7-4.4)

Cases: 174 male and 112 female colorectal cancer patients,

All(MV): $1.8(0.95-3.2)^{\mathrm{c}}$

University Hospital of Lausanne, Women ${ }^{(\mathrm{MV})}$ : $3.6(1.1-12.1)^{\mathrm{c}}$

Switzerland

Controls: 550 (269 men,

281 women) residents admitted to the same hospital for acute non-neoplastic conditions 
Table 1. (continued)

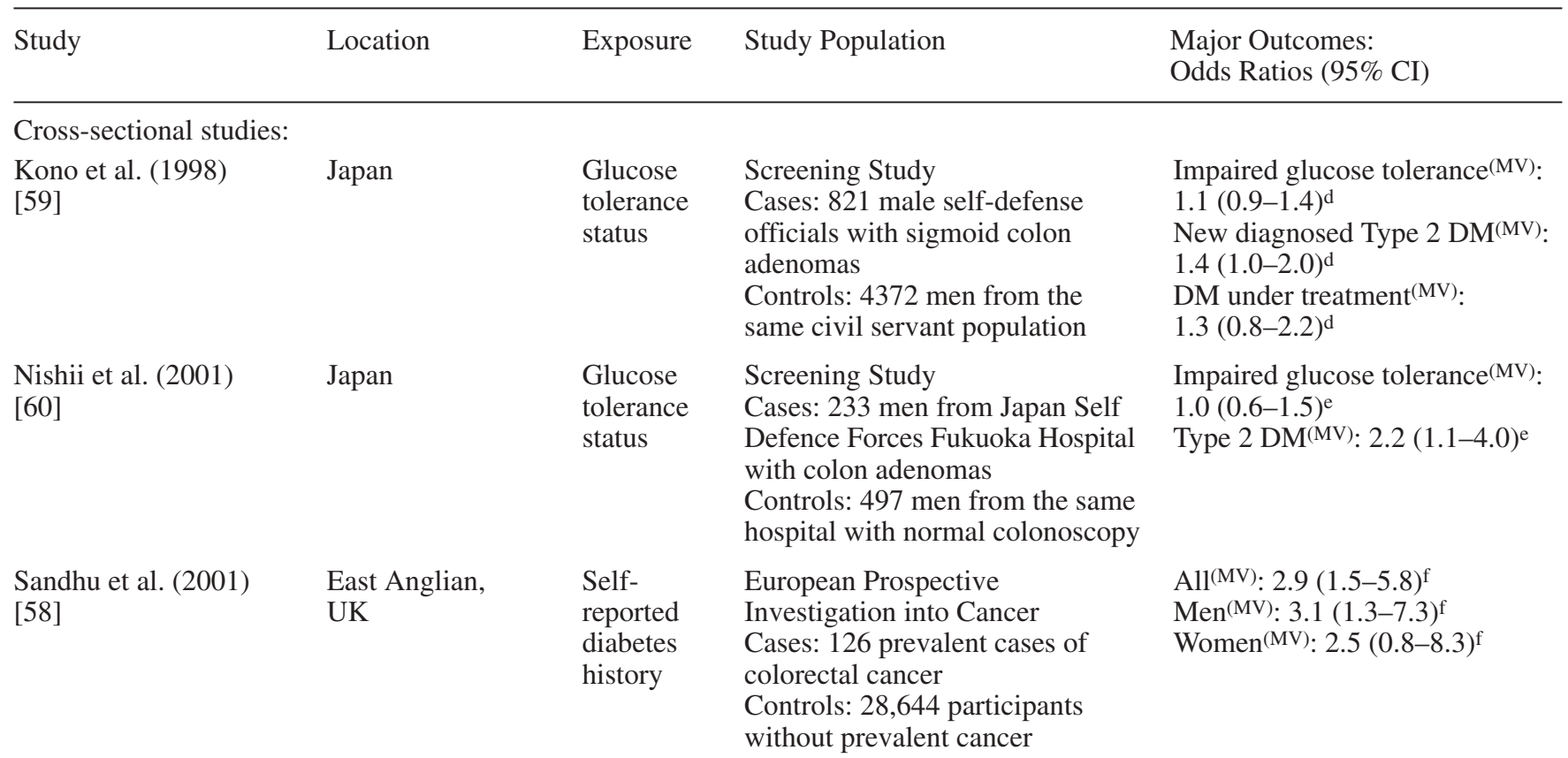

( ): Methods of adjustment; C: crude odds ratio, AS: age- or age/sex-adjusted odds ratio, and MV: Multivariate adjusted odds ratio

a Adjusted for age, sex, area of residence, education, BMI, and selected indicator foods (pasta or rice, meat, green vegetables, fresh fruits and coffee)

b Adjusted for age, sex, centre, education, body mass index, family history of colorectal cancer, physical activity, fat, total energy and fiber and alcohol intake

hyperglycaemia show increased risks, ranging from moderate to strong associations.

\section{Prospective studies}

In the past two decades, fifteen cohort studies, usually among diabetic patients, addressed the association between diabetes, hyperinsulinaemia, hyperglycaemia and colorectal cancer. Several of these studies were conducted in Nordic countries (Table 2).

Studies using cohorts of diabetic patients. The approach of using cohorts of diabetic patients and comparing the incidence in this group to the general population ensures a sufficient number of exposed individuals. A weakness of this design is the limited amount of data that can be collected from the comparison group (usually the general population), thus reducing the possibility to adjust for confounding factors beyond sex and age.

Most cohort studies of patients with diabetes reported moderately increased risks of colorectal cancer compared to the general population, with standardized incidence ratios (SIR, the standardized incidence ratio, is the ratio of total observed case number in the c Adjusted for age, smoking status, BMI, education, and alcohol consumption

d Adjusted for body mass index, cigarette smoking, alcohol use, rank of the Self Defence Forces, and hospital

e Adjusted for age and BMI

f Adjust for age, smoking status, BMI, education, and alcohol consumption

exposed group to the sum of expected case numbers, calculated by exposed persons in each specific age group multiplied by its compared age-specific incidence rate.) ranging from 1.0 to 1.4 (Table 2). The first cohort study was carried out in Rochester, Minnesota, USA among 1135 diabetic cases followed up for 25 years $(\mathrm{SIR}=1.2,95 \% \mathrm{CI}=0.7-1.8)$ [61]. However, the increased risk was only found among men. Several Nordic cohort studies reported similar findings $[62,63,64,65,66]$. For example, one study observed a significantly increased incidence of colon cancer $(\mathrm{SIR}=1.4,95 \% \mathrm{CI}=1.3-1.5)$ and rectal cancer ( $\mathrm{SIR}=1.2,95 \% \mathrm{CI}=1.1-1.4)$ among more than 150 000 population-based diabetic patients compared to the Swedish population, with a slightly higher excess risk for proximal and sigmoid colon cancers [65]. Wideroff et. al. followed more than 100000 subjects diagnosed with diabetes and observed a similarly increased risk for colon cancer among men $(\mathrm{SIR}=1.3$, 95\% CI $=1.1-1.4$ ), and a very slightly increased risk among women (SIR=1.1, 95\% CI=1.0-1.2) [66].

As discussed previously, possible uncontrolled confounding was a limitation for these studies $[61,62,63$, $64,65,66,67]$. Another restriction was the utilization of hospital-discharged diabetic patients as the exposed group $[63,65,66]$, which could have led to falsely 
Table 2. Prospective studies investigating associations between diabetes, hyperinsulinaemia, hyperglycaemia, and colorectal cancer (SIR, standardized incidence ratio; SMR, standardized mortality ratio; RR, relative risk)

\begin{tabular}{|c|c|c|c|}
\hline Study & Location & Exposure & Study Population \\
\hline
\end{tabular}

Cohorts of patients with diabetes

Ragozzino et al. (1982) Rochester, Initial diagnosis Exposed group: 1135 initially

[61]

\section{Minnesota, of diabetes mellitus*}

USA

(25-year follow-up)

Green et al. (1985)

[62]

Adami et al. (1991)

[63]

Uppsala, Sweden

Fyn County, Insulin-treated Denmark diabetes*

(8.5-year follow-up)

diagnosed diabetic patients

(120 cases)

Comparison group: Rochester

population

Exposed group: 783 male and

716 female insulin-treated

diabetic subjects (17 cases for

stomach, colon, and rectal cancer)

Comparison group:

Danish population

Smith et al. (1992)

[67]

London,

England

Hjalgrim et al. (1997) [64]

Funen

County,

Denmark

(37 cases for cancers for

Weiderpass et al. (1997) Sweden [65]

Wideroff et al. (1997) Denmark [66]

Discharge diagnosis
Exposed group: 23,146 male \& 27,862 female population-based diabetic patients (325 cases)

Comparison group: six-county Uppsala Healthy Care Region population

Previously diagnosed diabetes* (19-year follow-up)

Whitehall Study

Exposed group: 224 male civil

servants with a plasma glucose

level of $\geq 200 \mathrm{mg} / 100 \mathrm{ml}$ or with previously diagnosed diabetes (1 death from colon cancer) Comparison group: 17,051 male normoglycaemic civil servants

Insulin treated

diabetes*

Danish National Conscript

Registry

(19,511 person-years Exposed group: 1499 insulin of follow-up)

treated diabetic patients digestive organs and peritoneum combined)

Comparison group: Danish population

Discharge diagnosis of diabetes* (6 to 24-year follow-up)

Exposed group: 153,852 population-based diabetic patients (1,435 cases) Comparison group: Swedish population of diabetes* (628,129 person-

Danish Central Hospital Discharge Register Exposed group: 109,581 years of follow-up) nationwide subjects diagnosed with diabetes (1257 cases) Comparison group: Danish population

Male: $\mathrm{SIR}=1.2(0.9-1.4)$ for colon; $1.3(1.1-1.7)$ for rectum Female: $\mathrm{SIR}=1.0(0.8-1.2)$ for colon; 0.9 (0.7-1.2) for rectum

Diabetic men: SMR=0.6 (0.1-4.5) for colon cancer

Cancers for digestive organs and peritoneum combined:

$\mathrm{SIR}=1.3(0.6-2.6)$ for those diagnosed with diabetes before 30 years old:

SIR=1.1 (0.7-1.5) for those diagnosed with diabetes after age of 30

Male: $\mathrm{SIR}=1.4(1.2-1.5)$ for colon cancer; 1.4 (1.2-1.5) for rectal cancer

Female: SIR=1.4 (1.3-1.6) for colon cancer; $1.1(0.9-1.3)$ for rectal cancer

Males: SIR=1.3 (1.1-1.4) for colon cancer; $1.1(0.9-1.2)$ for rectal cancer

Females: SIR=1.1 (1.0-1.2) for colon cancer; 1.0 (0.9-1.2) for rectal cancer

Men: Adjusted RR=1.4 (0.6-3.3)

for colorectal cancer ${ }^{\mathrm{a}}$

National Health and Nutrition

Survey I (NHANES1) diabetes history* (15-year follow-up) diabetes at baseline (176 cases for colorectal cancer)

Comparison group: 12,532 nondiabetes reported NHANES1 subjects matching on race and follow-up time
(0.6-3.1) for colorectal cancer 
Table 2. (continued)

Study Location $\quad$ Exposure $\quad$ Study Population $\quad$ Major Outcomes (95\%CI)

\begin{tabular}{|c|c|}
\hline $\begin{array}{l}\text { Will et al. (1998) } \\
\text { [69] }\end{array}$ & USA \\
\hline
\end{tabular}

First Cancer Prevention Study of the American Cancer Society Exposed group: 7229 male and 8258 female volunteers diagnosed with diabetes (7224 cases) Comparison group: 848,212 recruited volunteers without diabetes

Hu et al. (1999) USA

[70]

USA Self-reported diabetes history** (18-year follow-up)

Nurses' Health Study

Exposed group: 7069 women

with history of diabetes (62 cases)

Comparison group:

111,003 women without history of diabetes

Nilsen et al. (2001)

Nord-

[71]

Trondelag county,

Diabetes history

Norway

(follow-up for

medium of

10.8 years)

Nord-Trondelag Health Survey Exposed group: 6510 personyears for men and 8202 personyears for women with history
Cohorts evaluating biomarkers of insulin-resistance

Smith et al. (1992)

[67]

London,

England

Impaired glucose

tolerance

(19-year follow-up) of diabetes ( 37 cases)

Comparison group: all residents in Nord-Trondelag county aged 20 years or older $(357,986$ and 376,715 person-years, respectively)

Whitehall Study

Exposed group: 999 male

nondiabetic civil servants with glucose concentrations above the $95^{\text {th }}$ centile ( 6 deaths from colon cancer)

Comparison group: 17,051 male normoglycaemic civil servants

Schoen et al. (1999) USA [72]

USA Fasting glucose, 2-hr glucose, Fasting insulin, and 2-hr insulin at baseline (follow-up for medium of 77 months)

Platz et al. (1999) USA

[73]

Glycosylated haemoglobin $\left(\mathrm{HbA}_{1 \mathrm{c}}\right)$ (follow-up for about 4 years)

Nilsen et al. (2001)

Nord-

[71]

Trondelag county, Norway
Cardiovascular Health Study Population-based cohort with 5888 subjects aged 65 years old or more (mostly minority groups, excluding treated diabetic subjects) (102 cases)

Nurses' Health Study Cases: 79 women with colorectal cancer Controls: 156 controls matched on year of birth, month of blood draw, and fasting state

Nord-Trondelag Health Survey Exposed group: 11,302 personyears for men and 6502 personyears for women with nonfasting blood glucose level $\geq 8.0 \mathrm{mmol} / \mathrm{l}$ (46 cases) Comparison group: all residents in Nord-Trondelag county aged 20 years or older
Men: Adjusted RR=1.3 (1.0-1.7)

for colorectal cancer ${ }^{b}$

Women: Adjusted RR=1.2

(0.9-1.5) for colorectal cancerb

Adjusted RR=1.4 (1.1-1.9) ${ }^{\mathrm{c}}$

Men: SIR=0.7 (0.4-1.2) for colorectal cancer

Women: SIR=1.6 (1.0-2.3) for colorectal cancer

Men with impaired glucose tolerance: $\mathrm{SMR}=0.8(0.4-1.8)$ for colon cancer

Q4/Q1 of fasting glucose: $R R=1.8$ (1.0-3.1) for colorectal cancer;

Q4/Q1 of 2-hr glucose: RR=2.4 (1.2-4.7) for colorectal cancer; Q4/Q1 of fasting insulin: $R R=1.2$ (0.7-2.1) for colorectal cancer; Q4/Q1 of 2-hr insulin: $R R=2.0$ (1.0-3.8) for colorectal cancer.

Tertile3/tertile1 of $\mathrm{HbA}_{1 \mathrm{c}}$ : $\mathrm{OR}=1.2(0.6-2.7)$ for colorectal cancer

$\mathrm{OR}=4.6$ (1.2-17.1) for advanced colorectal cancer

Men: $S I R=0.9(0.6-1.4)$ for colorectal cancer Women: SIR=2.0 (1.3-3.0) for colorectal cancer 
Table 2. (continued)

\begin{tabular}{|c|c|c|c|c|}
\hline Study & Location & Exposure & Study Population & Major Outcomes (95\%CI) \\
\hline $\begin{array}{l}\text { Trevisan et al. (2001) } \\
\text { [74] }\end{array}$ & Italy & $\begin{array}{l}\text { Insulin resistance } \\
\text { (7-year follow-up) }\end{array}$ & $\begin{array}{l}\text { Risk Factor and Life Expectancy } \\
\text { Project } \\
\text { Exposed group: } 1185 \text { persons } \\
\text { with insulin resistance syndrome } \\
\text { ( } 6 \text { cases) } \\
\text { Comparison group: } \\
36,116 \text { persons without insulin } \\
\text { resistance syndrome ( } 48 \text { cases) }\end{array}$ & $\begin{array}{l}\text { Total: } \mathrm{RR}=3.0(1.3-7.0) \\
\text { for colorectal mortality } \\
\text { Male: } \mathrm{RR}=3.0(1.1-8.3) \text { for } \\
\text { colorectal mortality } \\
\text { Female: } \mathrm{RR}=2.7(0.6-12.5) \text { fo } \\
\text { colorectal mortality }\end{array}$ \\
\hline $\begin{array}{l}\text { Colangelo et al. (2002) } \\
\text { [75] }\end{array}$ & $\begin{array}{l}\text { Chicago, } \\
\text { USA }\end{array}$ & $\begin{array}{l}\text { Post-load plasma } \\
\text { glucose (PLG) level } \\
\text { (866,926 person- } \\
\text { years of follow-up) }\end{array}$ & $\begin{array}{l}\text { Chicago Heart Association } \\
\text { Detection } \\
\text { Project in Industry } \\
\text { Population-based cohort with } \\
20,433 \text { men and } 15,149 \text { women } \\
\text { without diabetes at baseline; } \\
\text { follow-up since } 1967 \\
\text { ( } 317 \text { colorectal cancer deaths) }\end{array}$ & $\begin{array}{l}\text { Men }(\mathrm{PLG}, \mathrm{mg} / \mathrm{dl}): \\
\leq 119: \mathrm{RR}=1 \text { (reference) } 1 \\
\text { 20-159: RR=1.1 (0.8-1.6) } \\
\text { 160-199: RR=0.8 }(0.5-1.3)^{\mathrm{d}} \\
\geq 200: \mathrm{RR}=1.5(0.9-2.4)^{\mathrm{d}} \\
\text { Women }(\mathrm{PLG}, \mathrm{mg} / \mathrm{dl}): \\
\leq 119: \mathrm{RR}=1(\text { reference) } \\
\text { 120-159: RR=1.8 }(1.1-2.8)^{\mathrm{d}} \\
160-199: \mathrm{RR}=1.4(0.8-2.4)^{\mathrm{d}} \\
\geq 200: \mathrm{RR}=1.9(1.04-3.6)^{\mathrm{d}}\end{array}$ \\
\hline
\end{tabular}

*Not discriminating between Type 1 and Type 2 diabetes mellitus (DM)

** Type 2 DM only

a Adjusted for age, BMI, smoking, alcohol, income, and recreational physical activity

${ }^{b}$ Exact controlling factors were not specified

low associations by including diabetic patients in the comparison group. Most of these studies did not distinguish between Type 1 and Type 2 diabetes, although the vast preponderance of patients would be Type 2 diabetes [61, 62, 63, 64, 65, 66, 67]. Types of diabetes could differ in etiology and exposure to internal or environmental factors of importance for initiation or promotion of colorectal cancer. The number of colon cancer cases in the exposed group was small in several studies [62, 64, 67]. However, most studies with at least 100 exposed cases reported consistently increased SIRs, ranging between 1.1-1.4 [61, 65, 66].

Cohort studies among the general population. Several prospective studies evaluated diabetes history as a risk factor among the general population, or included baseline measures for evidence of insulin resistance or diabetes (Table 2). Major strengths of this design are the ability to control for confounding factors and elimination of selection bias. Studies investigating the risk of colorectal cancer associated with a history of diabetes reported slightly increased relative risks (ranging from 1.2 to 1.4$)[68,69,70]$. These included NHANES1, the First Cancer Prevention Study of the American Cancer Society, and the Nurses' Health Study, in which a significantly elevated relative risk was observed (adjusted $\mathrm{RR}=1.4$, 95\% $\mathrm{CI}=1.1-1.9$ ). A Norwegian study reported an increased risk only among women, but not for men (women: SIR=1.6, 95\% CI=1.0-2.3; men: SIR $=0.7,95 \%$ CI $=0.4-1.2$ ) [71]. Overall, these well-designed cohort studies pro- c Adjusted for age, time period, BMI, smoking before age 30, menopausal status, multivitamin supplement use, alcohol consumption, average hours per week of moderate or vigorous activity, aspirin use, parental history of colorectal cancer, and red meat consumption

d Adjusted for age, race, education, and height

vide support for a slightly increased risk of colorectal cancer associated with a history of diabetes.

Cohorts evaluating biomarkers of insulin-resistance. Several prospective studies used surrogate indicators for hyperinsulinaemia or hyperglycaemia and assessed future colorectal cancer incidence or mortality (Table 2). Impaired glucose tolerance (the sub-clinical stage of Type 2 diabetes) was found to be a negative (but not significant) predictor of colon cancer deaths among men (Standardized mortality ratio: $\mathrm{SMR}=0.8$, 95\% CI=0.4-1.8) [67]. (The standardized mortality ratio (SMR) is equivalent to the SIR, but replaces the age-specific incidence rate by the age-specific mortality rate.) However, assessing colon cancer mortality rather than incidence can result in biased findings for etiology. Diabetes is not only a possible risk factor, but also an important determinant of survival for colorectal cancer cases.

The Cardiovascular Health Study provided the first direct evidence of the association between glucose/insulin concentration and colorectal cancer in the elderly (65 years old or more) [72]. 2-h post-challenge glucose concentration was shown to be a significant risk factor of colorectal cancer (Q4/Q1 RR=2.4, 95\% $\mathrm{CI}=1.2-4.7$, Q: quartile, i.e. 25\%), whereas fasting glucose (Q4/Q1 $\mathrm{RR}=1.8,95 \% \mathrm{CI}=1.0-3.1)$ and $2 \mathrm{~h}$ post-challenge insulin concentrations $(\mathrm{Q} 4 / \mathrm{Q} 1 \mathrm{RR}=2.0$, 95\% CI=1.0-3.8) were positively associated, with borderline statistical significance [72]. These findings were replicated by Nilsen et al. for women $(\mathrm{SIR}=2.0$, 
95\% $\mathrm{CI}=1.3-3.0)$, but not for men $(\mathrm{RR}=0.9,95 \%$ $\mathrm{CI}=0.6-1.4) \quad$ (Nord-Trondelag Health Survey) [71]. Subjects with abnormal insulin/glucose measurements could have been potentially Type 2 diabetic patients. A limitation of these studies was that they did not collect information about anti-diabetic treatment regimens during the follow-up period. A case-control study nested in the Nurses' Health Study with a follow-up period of about 4 years used measurements of $\mathrm{HbA}_{1 \mathrm{c}}$ as an indicator of previous 2-month glycaemia concentration (direct) and blood insulin concentration (indirect). Although the risk associated with overall colorectal cancer or adenomas was not significantly increased, they observed a substantially increased risk for advanced colorectal cancer $(\mathrm{OR}=4.6,95 \%$ $\mathrm{CI}=1.2-17.1$ ), which indicates that hyperinsulinaemia might foster the progression of colorectal cancer [73]. As discussed by the authors, a weakness of this study was that blood samples were not collected substantially prior to diagnoses of cases (1989-90 vs 1989-94); thus, $\mathrm{HbA}_{1 \mathrm{c}}$ might not have reflected glycaemia status prior to the incidence of colorectal cancer, and measures could have been affected in some cases by subclinical colorectal cancer. Besides, $\mathrm{HbA}_{1 \mathrm{c}}$ could be a problematic marker of the average blood insulin concentration because different antidiabetic agent interventions would result in different insulin concentrations with similar $\mathrm{HbA}_{1 \mathrm{c}}$ percentage.

"X syndrome" (a cluster of metabolic abnormalities associated to insulin resistance-serum triglycerides, HDL cholesterol, glucose, and blood pressure) was treated as the main risk factor for colorectal cancer mortality in a combined cohort. An approximate three-fold increased risk was observed with RRs of 3.0 (95\% CI=1.1-8.3), 2.7 (95\% CI=0.6-12.5), and $3.0(95 \% \mathrm{CI}=1.3-7.0)$ for men, women, and both sexes combined respectively [74]. A recent large cohort study utilized 1-h post-load plasma glucose concentrations as an indicator of hyperinsulinaemia [75]. Both men and women with the highest post-load plasma glucose concentrations $(>200 \mathrm{mg} / \mathrm{dl})$ were found at risk of somewhat greater colorectal cancer mortality (men: $\mathrm{RR}=1.5,95 \% \mathrm{CI}=0.9-2.4$, women: $\mathrm{RR}=1.9$, 95\% $\mathrm{CI}=1.04-3.6)$ than those with the lowest concentrations $(<120 \mathrm{mg} / \mathrm{dl})$. Major limitations in these two studies were the use of mortality rather than incidence as the end point and regardless of medicine interventions, but the findings provide evidence for a modest association between insulin resistance syndrome and colorectal cancer mortality.

\section{Results among specific sub-groups}

Considering a slightly increased risk associated with a history of diabetes, hyperinsulinaemia, or hyperglycaemia, the question arises whether there are any subgroups of patients that are at particular risk. Are the associations consistent across men and women or do they differ for different sites of the colorectal tract? Several studies explored stratification by sex, colorectal cancer site, exposure time period, age at diagnosis of cancer or diabetes, and type of diabetes mellitus.

Sex differences. Six studies reported an increased risk of colorectal cancer among male diabetic patients but not in women $[52,58,61,63,66,74]$, in contrast to five others showing higher risks of colorectal or colon cancer for women, rather than men $[53,56,57,71$, 75]. Several other studies did not report sex differences $[51,54,55,65,68,69,74,75]$. Therefore, there is currently no consistent evidence for a differential association between diabetes and colorectal cancer among men and women.

Site-specific associations. Among the studies investigating site-specific associations, no consistent patterns were observed. However, the case numbers were often small, limiting statistical power. For colon cancer, an increased risk was detected in four studies $[50,53,65$, $66]$, and similarly for rectal cancer $[50,53,55,63,65]$. Some studies suggest that proximal and distal colon tumours differ in their histopathologic characteristics, precursor lesions, and patterns of associated molecular alterations and could be considered different entities $[71,76]$, and some studies have reported risk estimates stratified by tumour site $[56,60,65,70]$. In two cohort studies $[65,70]$ and one cross-sectional study [60], higher relative risks were detected for proximal colon tumours than those of the distal colon. However, a case-control study in Hawaii reported a higher risk of cancers in the distal colon in both men and women [56]. Thus, the current evidence regarding differences by tumour location is inconsistent, but indicates possibly a stronger association for the proximal colon.

Timing and duration of exposure. Studies investigating the temporal effects of an association between diabetes mellitus and colorectal cancer can provide clues to the underlying molecular mechanisms about the effect of hyperinsulinaemia/hyperglycaemia to different stages of colonic carcinogenesis. Some studies reported stronger associations with a longer time period between diagnosis of diabetes and colorectal cancer. La Vecchia et al. observed significantly increased odds ratios for rectal cancer, but not colon cancer, in patients with diabetes for $\geq 10$ years compared to those diagnosed less than 10 years previously [55]. The risk for colorectal cancer was substantially increased for individuals diagnosed with diabetes for five or more years $(\mathrm{OR}=4.1,95 \% \mathrm{CI}=1.6-10.8)$ in Levi et al.'s study [57]. In the Nurses' Health Study, an increased risk of colorectal cancer was observed for patients with 11 to 15 years of diabetes history, but not for those with $\leq 10$ years [70]. The authors suggested as a possible explanation that patients with long-term Type 2 diabetes 
could be more likely to be hypoinsulinaemic over longer periods due to pancreatic beta-cell decay [15]. Nevertheless, a Swedish study reported significantly increased risks of colon cancer among diabetic patients, with SIRs ranging from 1.3 to 1.5 , independent from the time of follow-up (1-4 years, 5-9 years, and 10-24 years), but the risks decreased by time length of follow-up for rectal cancer (SIRs=1.29, 1.25, 1.10, respectively) [65].

Age at diagnoses of diabetes mellitus or colorectal cancer. The age of onset of diabetes mellitus provides information regarding the relative importance of Type 1 (early onset in life) versus Type 2 (generally late onset) diabetes in colon carcinogenesis. Differences in findings between these two subtypes of diabetes mellitus could provide evidence for the underlying biological mechanisms and timing of diabetes progression. Although the data are scarce, there is some indication that a diagnosis of diabetes at an older age is more likely to be a risk factor for colon or rectal cancer $[55,66]$. In the series of studies from Italy, only patients diagnosed with colon or colorectal cancer $>60$ years old show an increased risk associated with diabetes $[53,55]$. Thus, one could hypothesize, that Type 2 diabetes (and insulin resistance) is of greater relevance in colorectal carcinogenesis. Overall, no patterns with respect to the temporal sequence emerged, which might be attributable to small sample sizes, or the inherent difficulty of measuring the "onset" of the exposures of hyperinsulinaemia and hyperglycaemia.

\section{Challenges for investigating the association between diabetes, hyperinsulinaemia, hyperglycaemia, and colorectal cancer}

Treatment modalities for diabetes can modify the relationship between insulin and glucose concentration, and thus need to be considered when evaluating the association between diabetic status and colorectal cancer. Diabetic treatment strategies have been continuously improved over the past decades. For the majority of Type 1 diabetic treatments, subcutaneous insulin injections are necessary, usually in combination with a controlled diet, exercise, self-monitoring of blood glucose, and routine monitoring of glycosylated haemoglobin $\left(\mathrm{HbA}_{1 \mathrm{c}}\right)$, urine glucose and urine ketones [77]. Potential alternative treatments, (e.g. islet cell transplantation or gene therapy) are still under experimental investigation [78, 79]. Among Type 2 patients, diet therapy, behavioural weight control, exercise, oral medications, and, eventually, insulin replacement are utilized to control blood glucose concentrations [77]. Furthermore, a combination therapy of insulin and oral antidiabetic agents is becoming increasingly used [80]. One category of antidiabetic agents increase insulin secre- tion by enhancing exocytosis of insulin-containing secretory granules (sulfonylureas) or by increasing calcium influx on beta-cell membranes (meglitinides). The other increases the peripheral glucose uptake and utilization (biguanides), influences insulin receptor activities and hepatic glucose uptake (thiazolidinediones), or delays glucose absorption and lower postprandial hyperglycaemia (alpha-glucosidase inhibitors) [81]. Thus, the first category raises insulin concentrations, while the latter one could possibly decrease insulin concentrations.

Few studies to date have investigated the association between Type 1 diabetes and colon cancer risk. Researching the association between Type 1 diabetic patients and colorectal cancer is challenging, and statistical power is usually limited: the exposure is rare and occurs at an early age, thus requiring a long follow-up period in cohort studies. Among patients with Type 1 diabetes undergoing adequate treatment, insulin concentrations usually stay within a similar range as in healthy individuals, while glucose concentrations are increased $[7,82]$. Therefore, using a study population of Type 1 diabetic patients will not provide an adequate setting for testing the hypothesis of hyperinsulinaemia as a risk factor of colorectal cancer. However, comparing these patients to healthy individuals can provide information regarding the hypothesis on hyperglycaemia and colorectal cancer.

In contrast, among Type 2 diabetic patients, insulin concentrations depend on their stage of diabetes and the antidiabetic medications used. At the early stage of (untreated) Type 2 diabetes, fasting insulin concentrations are increased, representing insulin resistance. An increase in exercise, use of oral antidiabetic agents, and body weight loss would delay or even prevent the need for insulin injections [7, 83]. One can infer that insulin concentrations must be higher in Type 2 patients who are untreated or treated conservatively only by diet control or increased physical activity. Among patients taking oral antidiabetics, they would be higher in those treated with sulfonylureas or meglitinides (drugs with the mechanism of increasing blood insulin concentration) than in those treated with biguanides, thiazolidinediones, or alpha-glucosidase inhibitors (drugs with the mechanism of increasing sensitivity to insulin). Thus, treatment modalities might be important factors that could obscure associations, and need to be taken into account when addressing the research question of hyperinsulinaemia, hyperglycaemia, and colorectal cancer.

\section{Future research directions}

As illustrated above, the diagnosis of diabetes by itself is only a crude surrogate measure of hyperinsulinaemia because insulin concentrations mostly depend on the severity of diabetes and treatment modalities (es- 
pecially for Type 2 diabetic patients). Future studies should aim to obtain specific information on these modifying factors, and ideally use records of blood glucose tests obtained during routine outpatient visits of diabetic patients. These measures could be obtained either prospectively in a cohort of Type 2 diabetic patients (comparing patients with poor glucose control to the ones with appropriate controls), or retrospectively for patients enrolled in large health plans (comparing cases/controls about their diabetes history, treatments carried out, and results of glucose control) like the Baltimore Longitudinal Study of Aging (BLSA) [84]. $\mathrm{HbA}_{1 \mathrm{c}}$ measurements would provide additional information regarding treatment efficacy and thus the endogenous exposure to insulin and glucose $[77,85]$. We suggest using insight in the natural history of diabetes, its clinical course, and the management with oral agents with different mechanisms to refine current research methods. Meanwhile, there is emerging evidence that the long-term use of aspirin and non-steroidal anti-inflammatory drugs (NSAIDs) can prevent colorectal neoplasia. In addition to several well-designed retrospective and prospective studies $[86,87,88,89,90]$ and intervention studies among patients with familial adenomatous polyposis [91, 92], two recent secondary prevention trials of colorectal adenomas showed a reduction in polyp risk among aspirin users $[93,94]$. Since many diabetic patients may use aspirin or NSAIDs for pain relief or cardiovascular disease prevention, this factor needs to be taken into account in future studies. If the proportion of aspirin/NSAID users among diabetic patients was larger than in the general population, potential confounding could be introduced that would mask a truly higher risk of colon cancer associated with diabetes mellitus.

In conclusion, most epidemiological studies summarized in this review support a slightly increased risk of colorectal cancer associated with diabetes or indicators of insulin resistance. However, limitations inherent in the studies undertaken to date could have restricted our ability to understand the true association between diabetes and colorectal cancer, as well as the underlying molecular mechanisms. Misclassification due to crude exposure measurements may have biased the observed associations towards the null. We suggest utilizing information on the natural course of diabetes mellitus, its specific treatment modalities, and biomarkers of endogenous exposure to further elucidate the relationship between diabetes, hyperinsulinaemia, hyperglycaemia, and colorectal cancer.

Acknowledgements. The authors wish to thank Drs. S. Davis, J.D. Potter and W.-S. Yang for their valuable suggestions in preparing this review article.

\section{References}

1. Edwards BK, Howe HL, Ries LAG et al. (2002) Annual report to the nation on the status of cancer, 1973-1999, featuring implications of age and aging on U.S. cancer burden. Cancer 94:2766-2792

2. Parkin DM, Whelan SL, Ferlay J, Raymond L, Young J (1997) Cancer incidence in five continents 1997. IARC, Lyon, France

3. Potter JD (1999) Colorectal cancer: molecules and populations. J Natl Cancer Inst 91:916-932

4. American Diabetes Association (1998) Standards of medical care for patients with diabetes mellitus. Diabetes Care 21 [Suppl 1]:S23-31

5. Felig P, Baxter JD, Frohman LA (1995) Endocrinology and metabolism, 3rd edition. McGraw-Hill Inc, Health Professions Division, New York

6. LeRoith D (2002) Beta-cell dysfunction and insulin resistance in type 2 diabetes: role of metabolic and genetic abnormalities. Am J Med 113 [Suppl 6A]:3S-11S

7. LeRoith D, Taylor SI, Olefsky JM (1996) Diabetes Mellitus-a fundamental and clinical text. Lippincott-Raven, Philadelphia New York

8. Zimmet P, Alberti KG, Shaw J (2001) Global and societal implications of the diabetes epidemic. Nature 414:782-787

9. McKeown-Eyssen G (1994) Epidemiology of colorectal cancer revisited: are serum triglycerides and/or plasma glucose associated with risk? Cancer Epidemiol Biomarkers Prev 3:687-695

10. Giovannucci E (1995) Insulin and colon cancer. Cancer Causes Control 6:164-179

11. Bruce WR, Wolever TM, Giacca A (2000) Mechanisms linking diet and colorectal cancer: the possible role of insulin resistance. Nutr Cancer 37:19-26

12. Bruce WR, Giacca A, Medline A (2000) Possible mechanisms relating diet and risk of colon cancer. Cancer Epidemiol Biomarkers Prev 9:1271-1279

13. Strickler HD, Wylie-Rosett J, Rohan T et al. (2001) The relation of type 2 diabetes and cancer. Diabetes Technol Ther 3:263-274

14. Kim YI (1998) Diet, lifestyle, and colorectal cancer: is hyperinsulinemia the missing link? Nutr Rev 56:275-279

15. Giovannucci E (2001) Insulin, insulin-like growth factors and colon cancer: a review of the evidence. J Nutr 131:3109S-3120S

16. Koenuma M, Yamori T, Tsuruo T (1989) Insulin and insulin-like growth factor 1 stimulate proliferation of metastatic variants of colon carcinoma 26. Jpn J Cancer Res 80:51-58

17. Watkins LF, Lewis LR, Levine AE (1990) Characterization of the synergistic effect of insulin and transferrin and the regulation of their receptors on a human colon carcinoma cell line. Int J Cancer 45:372-375

18. Bjork J, Nilsson J, Hultcrantz R, Johansson C (1993) Growth-regulatory effects of sensory neuropeptides, epidermal growth factor, insulin, and somatostatin on the nontransformed intestinal epithelial cell line IEC-6 and the colon cancer cell line HT 29. Scand J Gastroenterol 28:879884

19. Aaronson SA (1991) Growth factors and cancer. Science 254:1146-1153

20. MacDonald RS, Thornton WH Jr, Bean TL (1993) Insulin and IGE-1 receptors in a human intestinal adenocarcinoma cell line (CACO-2): regulation of $\mathrm{Na}$ glucose transport across the brush border. J Recept Res 13:1093-1113

21. Guo YS, Narayan S, Yallampalli C, Singh P (1992) Characterization of insulinlike growth factor I receptors in human colon cancer. Gastroenterology 102:1101-1108 
22. Warren RS, Yuan H, Matli MR, Ferrara N, Donner DB (1996) Induction of vascular endothelial growth factor by insulin-like growth factor 1 in colorectal carcinoma. J Biol Chem 271:29483-29488

23. Tricoli JV, Rall LB, Karakousis CP et al. (1986) Enhanced levels of insulin-like growth factor messenger RNA in human colon carcinomas and liposarcomas. Cancer Res 46:6169-6173

24. Lambert S, Collette J, Gillis J, Franchimont P, Desaive C, Gol-Winkler R (1991) Tumor IGF-II content in a patient with a colon adenocarcinoma correlates with abnormal expression of the gene. Int J Cancer 48:826-830

25. Culouscou JM, Remacle-Bonnet M, Garrouste F, Fantini J, Marvaldi J, Pommier G (1990) Production of insulin-like growth factor II (IGF-II) and different forms of IGF-binding proteins by HT-29 human colon carcinoma cell line. J Cell Physiol 143:405-415

26. Burgering BM, Medema RH, Maassen JA et al. (1991) Insulin stimulation of gene expression mediated by $\mathrm{p} 21$ ras activation. Embo J 10:1103-1109

27. Taha C, Klip A (1999) The insulin signaling pathway. J Membr Biol 169:1-12

28. Jhun BH, Meinkoth JL, Leitner JW, Draznin B, Olefsky JM (1994) Insulin and insulin-like growth factor-I signal transduction requires p21ras. J Biol Chem 269:5699-5704

29. Bos JL (1988) The ras gene family and human carcinogenesis. Mutat Res 195:255-271

30. Ma J, Pollak MN, Giovannucci E et al. (1999) Prospective study of colorectal cancer risk in men and plasma levels of insulin-like growth factor (IGF)-I and IGF-binding protein3. J Natl Cancer Inst 91:620-625

31. Kaaks R, Toniolo P, Akhmedkhanov A et al. (2000) Serum C-peptide, insulin-like growth factor (IGF)-I, IGF-binding proteins, and colorectal cancer risk in women. J Natl Cancer Inst 92:1592-1600

32. Giovannucci E, Pollak MN, Platz EA et al. (2000) A prospective study of plasma insulin-like growth factor-1 and binding protein-3 and risk of colorectal neoplasia in women. Cancer Epidemiol Biomarkers Prev 9:345-349

33. Yang R, Arem R, Chan L (1984) Gastrointestinal tract complications of diabetes mellitus. Pathophysiology and management. Arch Intern Med 144:1251-1256

34. Committee on Diet and Health (1989) Diet and health: implication for reducing chronic disease risk. National Academy Press, Washington DC

35. Committee on Diet, Nutrition and Cancer (1982) Diet, Nutrition and Cancer. National Academy Press, Washington DC

36. Stadler J, Yeung KS, Furrer R, Marcon N, Himal HS, Bruce WR (1988) Proliferative activity of rectal mucosa and soluble fecal bile acids in patients with normal colons and in patients with colonic polyps or cancer. Cancer Lett 38:315-320

37. Rafter JJ, Child P, Anderson AM, Alder R, Eng V, Bruce WR (1987) Cellular toxicity of fecal water depends on diet. Am J Clin Nutr 45:559-563

38. Rafter JJ, Eng VW, Furrer R, Medline A, Bruce WR (1986) Effects of calcium and $\mathrm{pH}$ on the mucosal damage produced by deoxycholic acid in the rat colon. Gut 27:1320-1329

39. Dukas L, Willett WC, Colditz GA, Fuchs CS, Rosner B, Giovannucci EL (2000) Prospective study of bowel movement, laxative use, and risk of colorectal cancer among women. Am J Epidemiol 151:958-964

40. Dukas L, Platz EA, Colditz GA, Willet WC, Giovannucci EL (2000) Bowel movement, use of laxatives and risk of colorectal adenomatous polyps among women (United States). Cancer Causes Control 11:907-914
41. Roediger WE (1980) Role of anaerobic bacteria in the metabolic welfare of the colonic mucosa in man. Gut 21:793-798

42. Jass JR (1985) Diet, butyric acid and differentiation of gastrointestinal tract tumours. Med Hypotheses 18:113118

43. Stamp D, Zhang XM, Medline A, Bruce WR, Archer MC (1993) Sucrose enhancement of the early steps of colon carcinogenesis in mice. Carcinogenesis 14:777-779

44. Caderni G, Bianchini F, Mancina A, Spagnesi MT, Dolara P (1991) Effect of dietary carbohydrates on the growth of dysplastic crypt foci in the colon of rats treated with 1,2-dimethylhydrazine. Cancer Res 51:3721-3725

45. Poulsen M, Molck AM, Thorup I, Breinholt V, Meyer O (2001) The influence of simple sugars and starch given during pre- or post- initiation on aberrant crypt foci in rat colon. Cancer Lett 167:135-143

46. Zhang XM, Chan CC, Stamp D, Minkin S, Archer MC, Bruce WR (1993) Initiation and promotion of colonic aberrant crypt foci in rats by 5- hydroxymethyl-2-furaldehyde in thermolyzed sucrose. Carcinogenesis 14:773-775

47. Franceschi S, Dal Maso L, Augustin L et al. (2001) Dietary glycemic load and colorectal cancer risk. Ann Oncol 12:173-178

48. Yamada K, Araki S, Tamura M et al. (1998) Relation of serum total cholesterol, serum triglycerides and fasting plasma glucose to colorectal carcinoma in situ. Int J Epidemiol 27:794-798

49. Bostick RM, Potter JD, Kushi LH et al. (1994) Sugar, meat, and fat intake, and non-dietary risk factors for colon cancer incidence in Iowa women (United States). Cancer Causes Control 5:38-52

50. Williams JC, Walsh DA, Jackson JF (1984) Colon carcinoma and diabetes mellitus. Cancer 54:3070-3071

51. O'Mara BA, Byers T, Schoenfeld E (1985) Diabetes mellitus and cancer risk: a multisite case-control study. J Chronic Dis 38:435-441

52. Kune GA, Kune S, Watson LF (1988) Colorectal cancer risk, chronic illnesses, operations, and medications: case control results from the Melbourne Colorectal Cancer Study. Cancer Res 48:4399-4404

53. La Vecchia C, D’Avanzo B, Negri E, Franceschi S (1991) History of selected diseases and the risk of colorectal cancer. Eur J Cancer 27:582-586

54. La Vecchia C, Negri E, Franceschi S, D’Avanzo B, Boyle P (1994) A case-control study of diabetes mellitus and cancer risk. Br J Cancer 70:950-953

55. La Vecchia C, Negri E, Decarli A, Franceschi S (1997) Diabetes mellitus and colorectal cancer risk. Cancer Epidemiol Biomarkers Prev 6:1007-1010

56. Le Marchand L, Wilkens LR, Kolonel LN, Hankin JH, Lyu LC (1997) Associations of sedentary lifestyle, obesity, smoking, alcohol use, and diabetes with the risk of colorectal cancer. Cancer Res 57:4787-4794

57. Levi F, Pasche C, Lucchini F, La Vecchia C (2002) Diabetes mellitus, family history, and colorectal cancer. J Epidemiol Community Health 56:479-480

58. Sandhu MS, Luben R, Khaw KT (2001) Self reported noninsulin dependent diabetes, family history, and risk of prevalent colorectal cancer: population based, cross sectional study. J Epidemiol Community Health 55:804-805

59. Kono S, Honjo S, Todoroki I et al. (1998) Glucose intolerance and adenomas of the sigmoid colon in Japanese men (Japan). Cancer Causes Control 9:441-446

60. Nishii T, Kono S, Abe H et al. (2001) Glucose intolerance, plasma insulin levels, and colon adenomas in Japanese men. Jpn J Cancer Res 92:836-840 
61. Ragozzino M, Melton LJd, Chu CP, Palumbo PJ (1982) Subsequent cancer risk in the incidence cohort of Rochester, Minnesota, residents with diabetes mellitus. J Chronic Dis 35:13-19

62. Green A, Jensen OM (1985) Frequency of cancer among insulin-treated diabetic patients in Denmark. Diabetologia 28:128-130

63. Adami HO, McLaughlin J, Ekbom A et al. (1991) Cancer risk in patients with diabetes mellitus. Cancer Causes Control 2:307-314

64. Hjalgrim H, Frisch M, Ekbom A, Kyvik KO, Melbye M, Green A (1997) Cancer and diabetes-a follow-up study of two population-based cohorts of diabetic patients. J Intern Med 241:471-475

65. Weiderpass E, Gridley G, Nyren O, Ekbom A, Persson I, Adami HO (1997) Diabetes mellitus and risk of large bowel cancer. J Natl Cancer Inst 89:660-661

66. Wideroff L, Gridley G, Mellemkjaer L et al. (1997) Cancer incidence in a population-based cohort of patients hospitalized with diabetes mellitus in Denmark. J Natl Cancer Inst 89:1360-1365

67. Smith GD, Egger M, Shipley MJ, Marmot MG (1992) Post-challenge glucose concentration, impaired glucose tolerance, diabetes, and cancer mortality in men. Am J Epidemiol 136:1110-1114

68. Steenland K, Nowlin S, Palu S (1995) Cancer incidence in the National Health and Nutrition Survey I. Follow-up data: diabetes, cholesterol, pulse and physical activity. Cancer Epidemiol Biomarkers Prev 4:807-811

69. Will JC, Galuska DA, Vinicor F, Calle EE (1998) Colorectal cancer: another complication of diabetes mellitus? Am J Epidemiol 147:816-825

70. Hu FB, Manson JE, Liu S et al. (1999) Prospective study of adult onset diabetes mellitus (type 2) and risk of colorectal cancer in women. J Natl Cancer Inst 91:542-547

71. Nilsen TI, Vatten LJ (2001) Prospective study of colorectal cancer risk and physical activity, diabetes, blood glucose and BMI: exploring the hyperinsulinaemia hypothesis. Br J Cancer 84:417-422

72. Schoen RE, Tangen CM, Kuller LH et al. (1999) Increased blood glucose and insulin, body size, and incident colorectal cancer. J Natl Cancer Inst 91:1147-1154

73. Platz EA, Hankinson SE, Rifai N, Colditz GA, Speizer FE, Giovannucci E (1999) Glycosylated hemoglobin and risk of colorectal cancer and adenoma (United States). Cancer Causes Control 10:379-386

74. Trevisan M, Liu J, Muti P, Misciagna G, Menotti A, Fucci F (2001) Markers of insulin resistance and colorectal cancer mortality. Cancer Epidemiol Biomarkers Prev 10:937-941

75. Colangelo LA, Gapstur SM, Gann PH, Dyer AR, Liu K (2002) Colorectal cancer mortality and factors related to the insulin resistance syndrome. Cancer Epidemiol Biomarkers Prev 11:385-391

76. Lindblom A (2001) Different mechanisms in the tumorigenesis of proximal and distal colon cancers. Curr Opin Oncol 13:63-69

77. DeFronzo RA (1998) Current therapy of diabetes mellitus. Mosby-Year Book, St. Louis, Missouri
78. Field LL (2002) Genetic linkage and association studies of Type I diabetes: challenges and rewards. Diabetologia 45:21-35

79. Bottino R, Trucco M, Balamurugan AN, Starzl TE (2002) Pancreas and islet cell transplantation. Best Pract Res Clin Gastroenterol 16:457-474

80. Taylor SI (1999) Current review of diabetes. Current Medicine, Philadelphia

81. (2000) Facts and comparisons. Drug Facts and Comparisons, St. Louis, Missouri

82. (1993) The effect of intensive treatment of diabetes on the development and progression of long-term complications in insulin-dependent diabetes mellitus. The Diabetes Control and Complications Trial Research Group. N Engl J Med 329:977-986

83. DeFronzo RA (2000) Pharmacologic therapy for type 2 diabetes mellitus. Ann Intern Med 133:73-74

84. Shock NW, Greulich RC, Andres R et al. (1984) Normal human aging: the Baltimore Longitudinal Study of Aging. NIH publication No. 84-2450. US Government Printing Office, Washington, DC

85. American Diabetes Association (2000) clinical practice recommendations 2000. Diabetes Care 23 [Suppl 1]:S1116

86. Garcia-Rodriguez LA, Huerta-Alvarez C (2001) Reduced risk of colorectal cancer among long-term users of aspirin and nonaspirin nonsteroidal antiinflammatory drugs. Epidemiology 12:88-93

87. Collet JP, Sharpe C, Belzile E, Boivin JF, Hanley J, Abenhaim L (1999) Colorectal cancer prevention by non-steroidal anti-inflammatory drugs: effects of dosage and timing. Br J Cancer 81:62-68

88. Giovannucci E, Rimm EB, Stampfer MJ, Colditz GA, Ascherio A, Willett WC (1994) Aspirin use and the risk for colorectal cancer and adenoma in male health professionals. Ann Intern Med 121:241-246

89. Giovannucci E, Egan KM, Hunter DJ et al. (1995) Aspirin and the risk of colorectal cancer in women. N Engl J Med 333:609-614

90. Smalley W, Ray WA, Daugherty J, Griffin MR (1999) Use of nonsteroidal anti-inflammatory drugs and incidence of colorectal cancer: a population-based study. Arch Intern Med 159:161-166

91. Steinbach G, Lynch PM, Phillips RK et al. (2000) The effect of celecoxib, a cyclooxygenase-2 inhibitor, in familial adenomatous polyposis. N Engl J Med 342:19461952

92. Giardiello FM, Hamilton SR, Krush AJ et al. (1993) Treatment of colonic and rectal adenomas with sulindac in familial adenomatous polyposis. N Engl J Med 328:13131316

93. Sandler RS, Halabi S, Baron JA et al. (2003) A randomized trial of aspirin to prevent colorectal adenomas in patients with previous colorectal cancer. N Engl J Med 348:883890

94. Baron JA, Cole BF, Sandler RS et al. (2003) A randomized trial of aspirin to prevent colorectal adenomas. N Engl J Med 348:891-899 